\title{
Minimum Electrical Energy Use in a Multi-Actuator System
}

DOI:

doi: 10.1109/IEMDC.2017.8002196

\section{Document Version}

Accepted author manuscript

Link to publication record in Manchester Research Explorer

\section{Citation for published version (APA):}

Apsley, J., Bodrov, A., Heath, W., \& Navarro Poupard, E. (2017). Minimum Electrical Energy Use in a MultiActuator System. In 2017 IEEE International Electric Machines \& Drives Conference (IEMDC), Miami IEEE. https://doi.org/doi: 10.1109/IEMDC.2017.8002196

\section{Published in:}

2017 IEEE International Electric Machines \& Drives Conference (IEMDC), Miami

\section{Citing this paper}

Please note that where the full-text provided on Manchester Research Explorer is the Author Accepted Manuscript or Proof version this may differ from the final Published version. If citing, it is advised that you check and use the publisher's definitive version.

\section{General rights}

Copyright and moral rights for the publications made accessible in the Research Explorer are retained by the authors and/or other copyright owners and it is a condition of accessing publications that users recognise and abide by the legal requirements associated with these rights.

\section{Takedown policy}

If you believe that this document breaches copyright please refer to the University of Manchester's Takedown Procedures [http://man.ac.uk/04Y6Bo] or contact uml.scholarlycommunications@manchester.ac.uk providing relevant details, so we can investigate your claim.

\section{OPEN ACCESS}




\title{
Minimum Electrical Energy Use in a Multi-Actuator System
}

\author{
Alexey Bodrov ${ }^{1}$, Eduardo Poupard ${ }^{2}$, William P. Heath ${ }^{2}$, Judith Apsley ${ }^{1}$ \\ ${ }^{1}$ Power Conversion Group, The University of Manchester, Manchester, M13 9PL, United Kingdom, \\ alexey.bodrov@manchester.ac.uk \\ ${ }^{2}$ Control Systems Centre, The University of Manchester, Manchester, M13 9PL, United Kingdom
}

\begin{abstract}
Many modern industrial systems include more than one electrical motor; for example, cutting tools and 3D printers include positioning systems of linear actuators driven by motors. Reducing energy consumption is an increasing priority for all sectors including manufacturing industries. Conventionally during the off-cut operation, positioning systems are controlled in either rapid positioning mode or linear interpolation mode. In both these modes, motors are controlled independently from each other, leading to increased energy consumption or a rise in operational time. In this paper, a novel method of energy use minimization by controlling drives with respect to each other is presented. It utilizes the Model Predictive Control (MPC) technique to identify the optimal state trajectory. This trajectory is used as feed-forward to the classical cascaded controller. For the particular case studied, experimental results show $13 \%$ and $8 \%$ efficiency rises compared to the rapid positioning and linear positioning modes respectively. The technique is validated on a dual brushed DC motor system.
\end{abstract}

Keywords-multi-drive systems; positioning systems; efficiency optimization; MPC

\section{INTRODUCTION}

Linear actuators, which convert a rotary motion into a linear motion, are widely used in modern industry. They are an important part of automation systems, where accurate motion control is required. Mainly due to their efficiency, flexibility and ease of being re-programmed, electric machines are used as the driving force of such actuators. This paper is focused on minimization of energy use by electric machines in systems with more than one actuator.

In literature many different approaches are suggested to improve the efficiency (minimization of energy usage) of electric motor drive systems. Some are focused on the motor design [1], others deal with inverter improvement either by applying advanced semiconductor devices [2], or modified inverter topologies [3], or modulation strategies [4]. Improving the motor drive efficiency by considering the operation of both the motor and the drive is reported as well [5]. However, Prof. J.W. Kolar, amongst others has defined one major challenge or opportunity for future improvement through systems-oriented analysis, where the study of multi-drive systems is believed to offer big potential for optimization. [6]

This research was supported by the University of Manchester President's Doctoral Scholarship Fund and the Engineering and Physical Sciences Research Council, Grant No. EP/K034987/1, Underpinning Power Electronics - Integrated Drives.
Positioning systems of milling machines, lathes, 3D printers or pick-and-place machines are a good example of multi-actuator systems. In these systems, drives perform a common task of moving a table or tool to the desired point in a 3D space, so axes are indirectly, mechanically coupled through the trajectory. In this work, it is assumed that the electric drives are comprised of equal motors connected through the separate convertors to the AC grid. This paper presents the minimum energy solution for systems of indirectly coupled drives from the perspective of motor control, where the overall system efficiency is a priority, rather than that of a single motor drive. Losses in the electrical machine depend on both current (torque) and frequency (speed), however for positioning applications, the machine operates mostly at low speed, so copper loss is assumed to be dominant. For the experimental verification of the proposed method, a dual brushed DC motor system representing the actuators of a two-axis system is built.

The paper is structured as follows. First, a typical machining center design is discussed and the need to improve efficiency is justified. Then the conventional approach to controlling the multi-actuator systems is described. The proposed method for minimizing the energy used, while keeping the overall performance at the same level is presented. The MATLAB simulation is shown, which supports the theoretical conclusions. Finally the experimental setup together with the experimental results are discussed.

\section{Motivation, SySTEM DESCRIPTION}

In Fig. 1 a diagram of typical positioning solution by FESTO is presented. Its working head is located next to the $z$ axis motor and can perform an effective work (drilling, cutting, handling, etc.). It can be moved in three different directions $x, y$ and $z$, however for the simplicity only $x$ - and $y$-axis movement is considered. According to [7], [8] the electrical energy consumed by a machining center can be split into three categories: idle operations, run-time operations (positioning and workpiece loading) and 'in-cut' operations (material removing). The split of energy between operations differs according to the machine design and use scenario, but in most cases material removal is not dominant and could be as small as $14.3 \%$ of overall energy usage. This compares with up to the $40 \%$ of overall energy for positioning operations, making it important to maximize the energy efficiency of positioning systems. In addition, it is preferable to complete the positioning task as soon as possible, due to the high background energy 


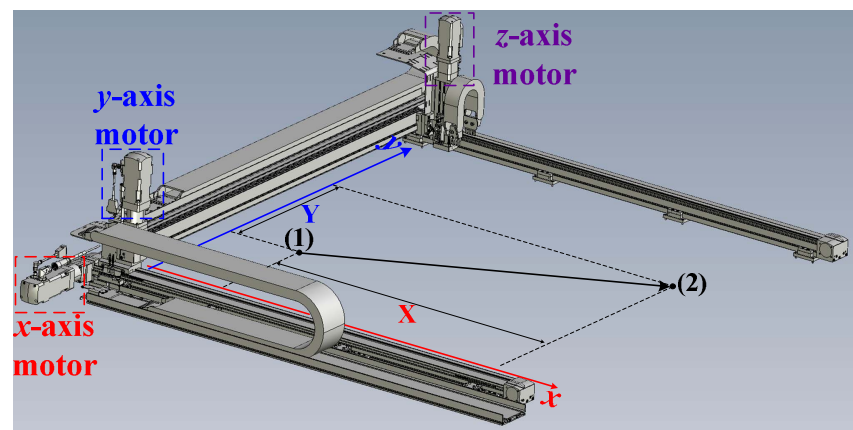

Fig. 1. Typical positioning system.

consumption of many uncontrolled auxiliary power consumers (such as coolant and oil pressure pumps) and the desire for increased production throughput.

This research proposes to minimise the 'off-cut' energy use of a positioning system comprised of two servo motors. Existing research optimizes the trajectory of the tool path or improves its accuracy [9],[10]. However, this matters only when the spindle motor is loaded, i.e. the machine tool is performing some action and cutting metal. Whilst cutting, the movement affects the quality of cut, which is beyond the scope of this research. During the overall operation of the system, for a significant portion of time the spindle motors are unloaded, while the machining centre performs some positioning job, for example moving the tool head from point (1) to point (2) where a new hole is going to be made or to the tool magazine. In this case, precise following of the linear trajectory is not required and it is possible to control the axis of movement with the longer path distance in a time-optimal way, while the second axis, with a shorter required path, could be controlled in an energy efficient way. If the proposed control strategy is implemented the overall operation time would not be changed (or even reduced if motors were not previously controlled in a time optimal way), however the overall energy consumption would be reduced.

For simplicity only a 2D positioning system is discussed, however the proposed strategy could be easily extended to 3D systems. In that case the axis with the longest required path is controlled in a time optimal way, whereas the two others are controlled in an energy optimal way.

\section{CNC MOVE PROFILES}

In $\mathrm{CNC}$ milling machines, during the off-cut operation, two different approaches (known as G-codes for FANUC and similar systems [11]) are adopted to control the 2D positioning system movement. G00 is a rapid positioning mode, where both $x$ - and $y$-axis motors are controlled at their maximum capability, which yields different end times of operation for each drive. G01 is a linear interpolation mode, where the drives are controlled to follow a linear trajectory connecting the $2 \mathrm{D}$ start and end points.

In order to control a single drive within the G00 or G01 modes, servo drives are controlled in a cascaded manner, where the position reference is obtained by applying one of the trajectories widely presented in literature [12]. In order to

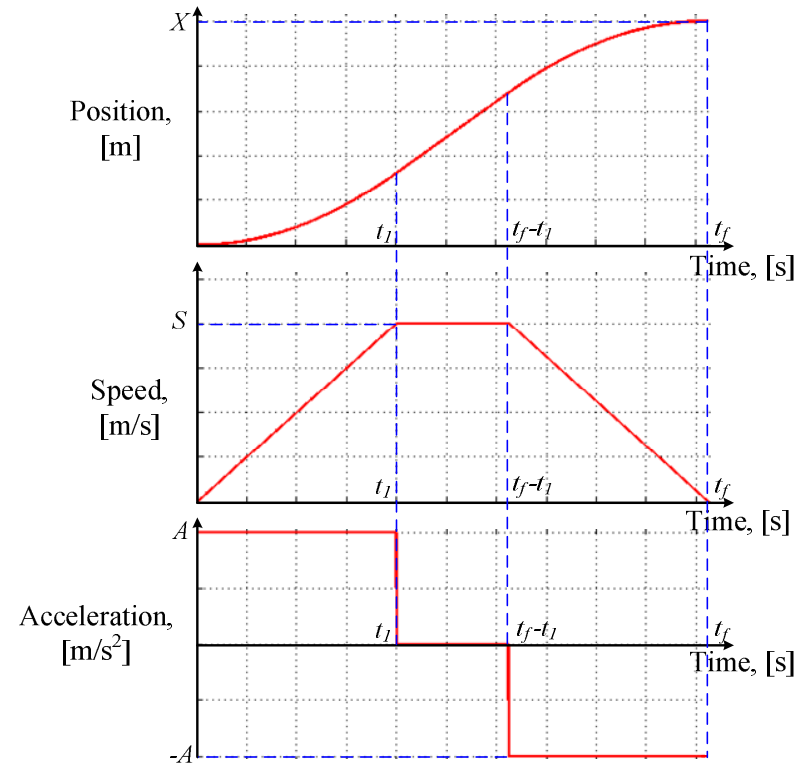

Fig. 2. Trapezoidal velocity profile.

enhance the performance of the motor drive, speed and acceleration references are fed forward to precisely tuned speed and current PI controllers respectively. Probably the most common position profile is the so-called "trapezoidal velocity", which is shown on Fig. 2, where the maximum speed and acceleration are limited to the values denoted by $S$ and $A$ respectively.

According to [13], control requirements for a positioning drive are limited to fast response time, zero overshoot, smooth transient response and minimum steady state error, but these can conflict, so the importance of each depends on the application. This is done by precise tuning of a servo motor PI control system, which is designed to follow the sophisticated speed profile.

When drives are controlled in G00 mode with a trapezoidal velocity profile, the end point of a trajectory is reached by the tool in minimum time, but this results in highly non-linear spindle motion and the highest energy consumption. When drives are controlled in G01 mode, the end point is reached more slowly, due to the reduction of the maximum speed limit (feed rate). Due to this, a new way to control the drives in a positioning system is proposed, which should be as fast as G00 and keep the loss at a minimum.

\section{Proposed Control Strategy}

In the development of $\mathrm{CNC}$ machines, the motors driving the linear actuators are frequently analysed only from the point of view of their mechanical characteristics [12], where the whole system is represented by a double integration between position and acceleration. With such a simplified representation, current control loop dynamics are not taken in account and limits on maximum speed and acceleration do not accurately reflect maximum current and voltage restrictions imposed on motor drives. In addition, the load is treated as mainly inertial, neglecting friction. 
In order to overcome the aforementioned drawbacks a novel control method is presented. Differential equations describing the dynamics of a machine are shown in (1). The brushed DC motor is used for simplicity.

$$
\begin{cases}d i_{a} / d t=-i_{a} R_{a} / L_{a}-\omega_{r m} \lambda_{f} / L_{a}+v_{a} / L_{a} & \left|i_{a}\right| \leq I_{\max } \\ d \omega_{m} / d t=i_{a} \lambda_{f} / J_{e}-\omega_{r m} B_{f r} / J_{e} & \left|v_{a}\right| \leq V_{\max } \\ d \theta_{r m} / d t=\omega_{r m} & \end{cases}
$$

where $i_{a}$ and $v_{a}$ are the armature current and voltage, $\lambda_{f}$ is the back EMF constant, $\theta_{r m}$ and $\omega_{r m}$ are rotor position and speed, $J_{e}$ and $B_{f r}$ are the rotor inertia and friction coefficient respectively. $I_{\max }$ and $V_{\max }$ represent the inverter maximum current and voltage. Given the linear model of DC machine (1) and introducing a set of state variables $\boldsymbol{x}^{T}=\left[x_{1}, x_{2}, x_{3}\right]^{T}=\left[i_{a}, \omega_{r m}, \theta_{r m}\right]^{T}$ with a control $u=v_{a}$, the problem is formulated in Table I. The description is the same for both motors, except the reference final position, where for the $x$-axis motor it equals to $X$, and for $y$-axis motor, $Y$. If distance $X$ is bigger than $Y$ then the $x$-axis actuator is controlled in a time optimal way and the $y$-axis actuator in an energy optimal way using the final time $\left(t_{f}\right)$ from the $x$-axis motor operation and vice versa.

To deal with final-time constrained optimization problems Model Predictive Control (MPC) is presented in the literature [14],[15], which solves an optimization problem every sampling time in a receding-horizon fashion. In Table I, two, problems are introduced, optimal time and optimal energy. Each requires special MPC formulation and solution. The procedure to solve the energy optimization problem is presented, considering that the final process time $t_{f}$ is already available from the optimal time problem.

Because of the domination of copper loss, in the energy efficiency problem formulation the square of the armature current was chosen as a performance criterion. Due to this, socalled Linear MPC which deals with a quadratic performance index, a linear system model (equality constraints) and linear inequalities (boundaries on states and inputs) is an appropriate choice. The aim of the general quadratic programming (QP) problem is to find a decision vector $\theta=\left[x_{0}^{T}, \ldots, x_{N}^{T} ; u_{0}^{T}, \ldots, u_{N-1}^{T}\right]^{T}$ which minimizes the quadratic performance index, subject to the constraints. The task is to express the problem formulated in Table I into the MPC form, which further should be expanded to the form of the QP problem and solved numerically. Sparse MPC formulation is adopted.

For the optimal energy control, the problem is defined as follows. Given an initial state $x_{0}$, compute a finite-horizon, state variable sequence $\chi=\left(x_{0}, x_{1}, \ldots, x_{N}\right)$, and an input sequence $U=\left(u_{0}, u_{1}, \ldots, u_{N-1}\right)$, which minimize the quadratic performance index while guaranteeing that all the constraints (equalities and inequalities) are satisfied over the prediction horizon $i \in 0,1 \ldots, N$ for a prediction horizon $N$ corresponding to the process final time $t_{f}$. In addition, final position and speed are specified for the end of horizon $N$. The mathematical definition of MPC is as follows.
TABLE I. PROBLEM FormULATION

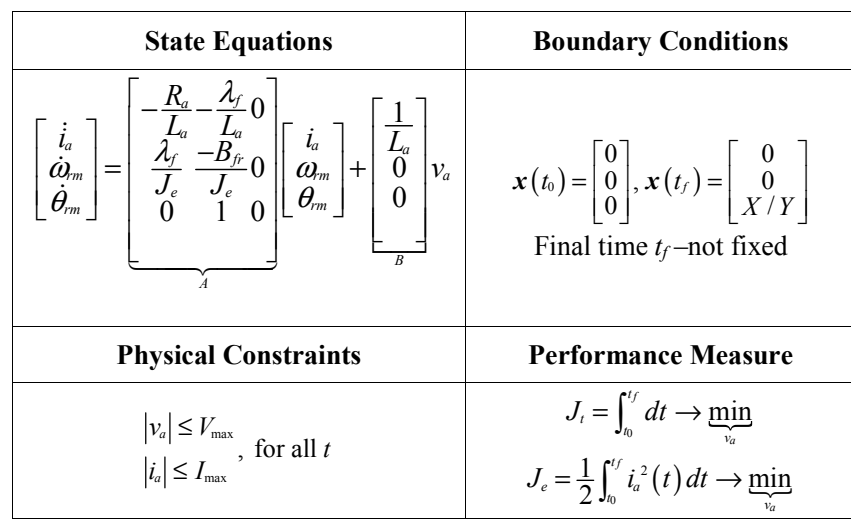

$$
\begin{gathered}
\min _{\chi, U} x_{N}^{T} P x_{N}+\sum_{i=0}^{N-1}\left(x_{i}^{T} Q x_{i}+u_{i}^{T} R u_{i}\right) \\
\text { subject to: } \begin{array}{cc}
x_{i+1}=A x_{i}+B u_{i} & x_{\text {low }} \leq x_{i} \leq x_{\text {high }} \\
I_{N} x_{N}=s_{N} & u_{\text {low }} \leq u_{i} \leq u_{\text {high }}
\end{array}
\end{gathered}
$$

where $A$ and $B$ are constant state matrices defined in Table I and $s_{N}$ contains the end constraints of the states at horizon $N$ $\left(\boldsymbol{x}\left(t_{f}\right)\right)$. Affine inequality constraints represent the system physical limitations and $P, Q$ and $R$ are the performance index weighting parameters for the final state, and the sequence of states and inputs respectively. $P$ and $Q$ are positive semidefinite while $R$ is positive definite and symmetric. To reflect the copper loss domination in the performance index the first element of matrices $P$ and $Q$, relating to $x_{1}=i_{a}$ has the highest weighting.

The MPC formulation in (2) can be expressed as a quadratic problem defined as

$$
\begin{aligned}
& \min _{\theta} f(\theta)=1 / 2 \theta^{T} H \theta+g^{T} \theta \\
& \text { subject to: } f \theta=b ; C \theta \leq d
\end{aligned},
$$

where

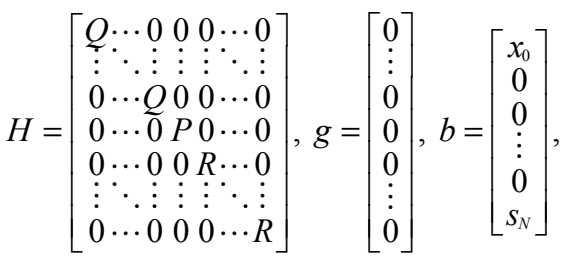

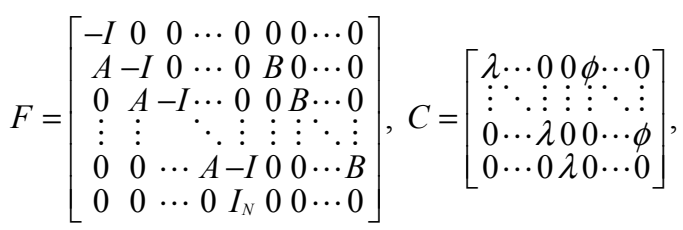

$$
\begin{aligned}
& d=\left[\begin{array}{c}
\xi \\
\vdots \\
\xi \\
\xi
\end{array}\right], \quad \begin{array}{c}
\lambda=[-I I 00]^{T} \\
\phi=\left[-x_{\text {low }} x_{\text {high }}-u_{\text {low }} u_{\text {high }}\right]^{T}
\end{array}
\end{aligned}
$$




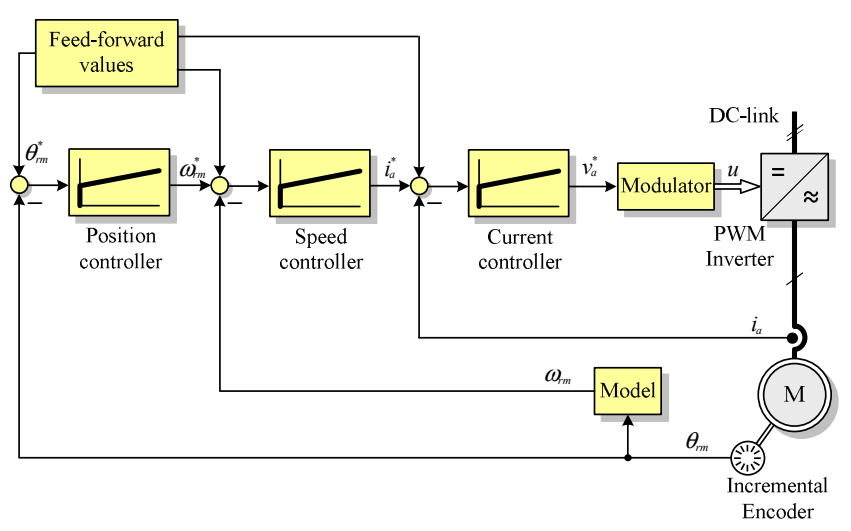

Fig. 3. Cascaded motor control.

and $I$ is an identity matrix. After the substitution of (3) to QP problem formulation the open-loop optimal energy control solution for the problem of moving one actuator to the required distance by a specific time $\left(t_{f}\right)$ can be solved numerically by any standard numerical quadratic program solver such as quadprog. $m$ in MATLAB. The result of these calculations would be the optimal control vector and reference trajectory.

The optimal time problem is solved by applying the procedure described in [16] and called Time Optimal MPC (TOMPC). In TOMPC the optimization problem is formulated in the same way as (2) with some arbitrary prediction horizon $N$. The feasibility of the solution is checked; if feasible, the prediction horizon is reduced and procedure repeats. After finding the unfeasible solution, the last feasible one is considered as time optimal; control and state reference trajectories are saved and last feasible $N$ is substituted to the energy optimal problem.

The open-loop solutions given above cannot be directly used in practice due to motor parameter variations and unpredictable external influences. So the drives should be forced to follow the predefined reference trajectories [17]. This is achieved by feed-forwarding the MPC-calculated states to the conventional cascaded controller shown if Fig. 3. Feedforwarding of the MPC-calculated states makes it possible to find the QP solution of-line, so on-line computational effort is profoundly reduced.

\section{SimULATION RESULTS}

The proposed control method of the multi-motor system was checked by MATLAB simulation. Rapid positioning mode G00, linear interpolation mode G01 and the proposed one were compared according to the energy consumption for the case where the $x$-axis distance was $30 \%$ longer than the $y$ - one $(9$

TABLE II. DC MOTOR PARAMETERS M642TE(150[W])

\begin{tabular}{|c|l|l|l|}
\hline $\boldsymbol{R}_{a}[\Omega]$ & 0.8 & $\boldsymbol{J}_{\boldsymbol{e}}\left[\mathbf{k g} \cdot \mathbf{m}^{2}\right]$ & $14 \mathrm{e}-4$ \\
\hline $\boldsymbol{L}_{\boldsymbol{a}}[\mathbf{m H}]$ & 0.42 & $\boldsymbol{V}_{\max }[\mathbf{V}]$ & 24 \\
\hline$\lambda_{\boldsymbol{f}}[\mathbf{V} /(\mathbf{r a d} / \mathbf{s})]$ & 0.095 & $\boldsymbol{I}_{\max }[\mathbf{A}]$ & 8 \\
\hline
\end{tabular}

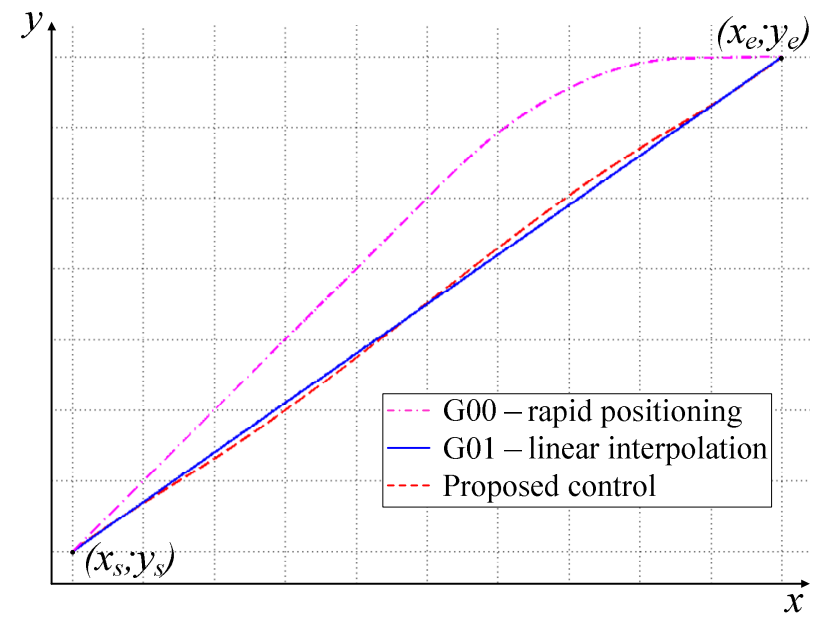

Fig. 4. Tool trajectories.

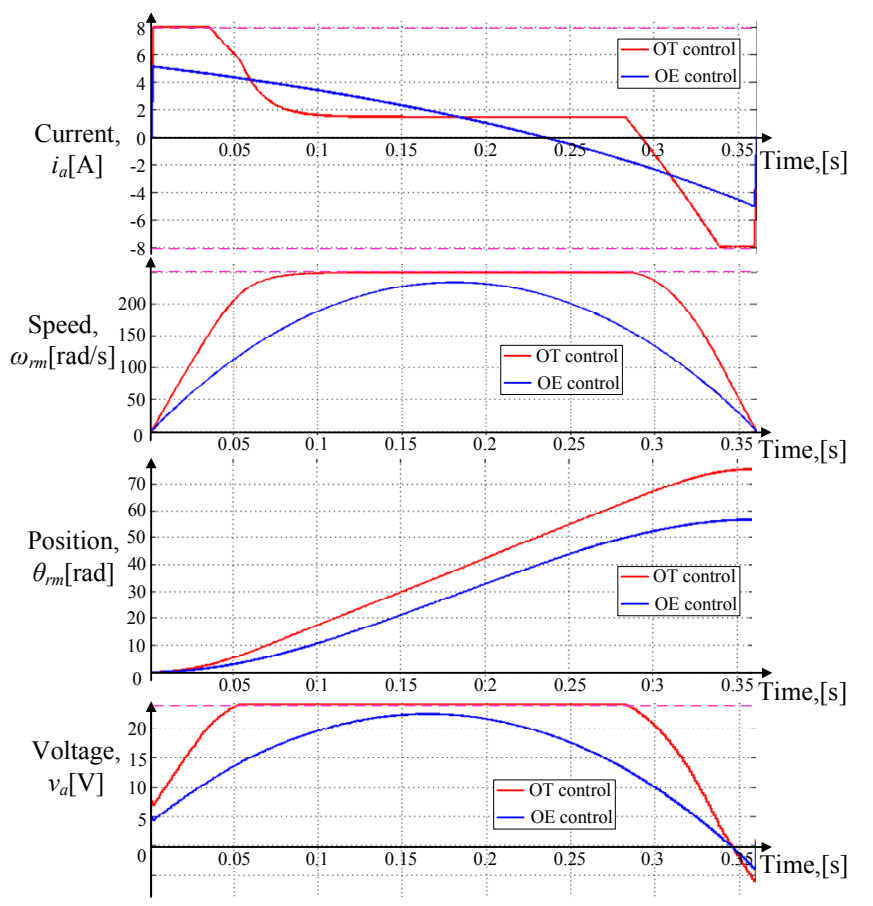

Fig. 5. Simulated state and control variables trajectories.

and 12 motor shaft rotations respectively). The conventional resultant tool trajectories together with the one obtained by the proposed method are shown in Fig. 4. The proposed trajectory is fairly close to G01, whereas the G00 mode control yields to a so-called "hockey-stick" motion, which could be a problem for obstacle avoidance. Experimental setup parameters presented in Table II are used for the simulation. For all three cases the motor was controlled by a $10 \mathrm{kHz}$ switching inverter and trapezoidal velocity profiles were assumed for drives in G00 and G01 modes. The maximum drive speed was set as $S=V_{\max } / \lambda_{f}$ and maximum acceleration was set as $A=\lambda_{f} I_{\max } / J_{e}$. To make a fair comparison, the feed rate for the drive with a longer path in the G01 mode was kept on its maximum level. Feed-forward values for all three modes were 
used for the cascaded controller shown on Fig. 3. PD-P-PI controllers for position, speed and current respectively have been chosen. The differential component is added to the position controller in order to supress the vibration close to the set point value. The respective bandwidths are $3.5 \mathrm{~Hz}, 35 \mathrm{~Hz}$ and $350 \mathrm{~Hz}$. In order to make a fair comparison, gain tuning is the same for all modes of operation.

Simulation shows that for the given example, when $x$-axis path is equal to the 12 rotations and controlled in optimal time way (OT) and $y$-axis path is 9 rotations and controlled in optimal energy way (OE), the net energy consumed by both motor drives controlled in the proposed way is reduced by around $25 \%$ compared to mode G00 and 14\% compare to G01. The simulation state and control trajectories of both drives controlled in the proposed way are shown on Fig. 5. Both drives fulfil the task in 0.36s and their states do not exceed the maximum values, however the armature current of $y$-axis has lower peak magnitude and smoother current variation, making the system copper loss lower.

\section{EXPERIMENTAL RESULTS}

To verify the obtained results experimentally, the experimental setup for one of the motor drives was assembled. The appearance is shown in Fig. 7. Motors are controlled using a commercially-available Texas Instruments board equipped with a digital controller based on the TMS320F28379D DSP and PS21765 intelligent power module. The board is powered through the external DC power supply. In order to measure the electrical energy consumption a Yokogawa WT3000 power

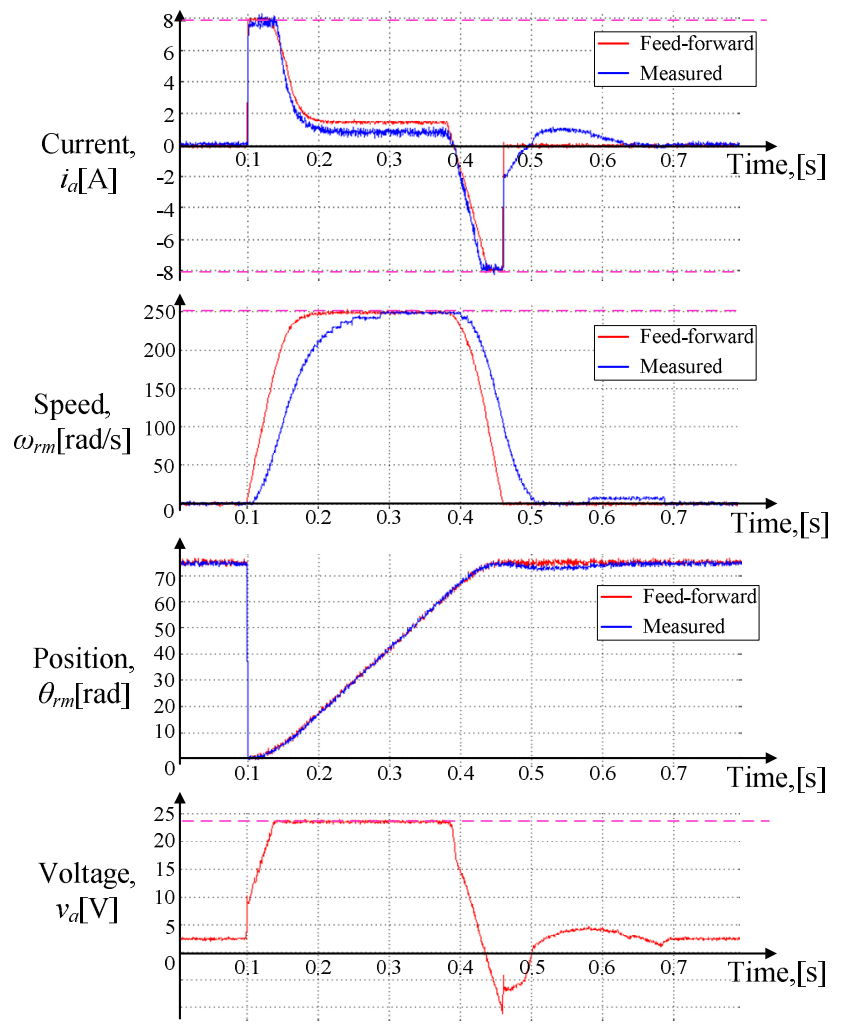

Fig. 6. Optimal time control.

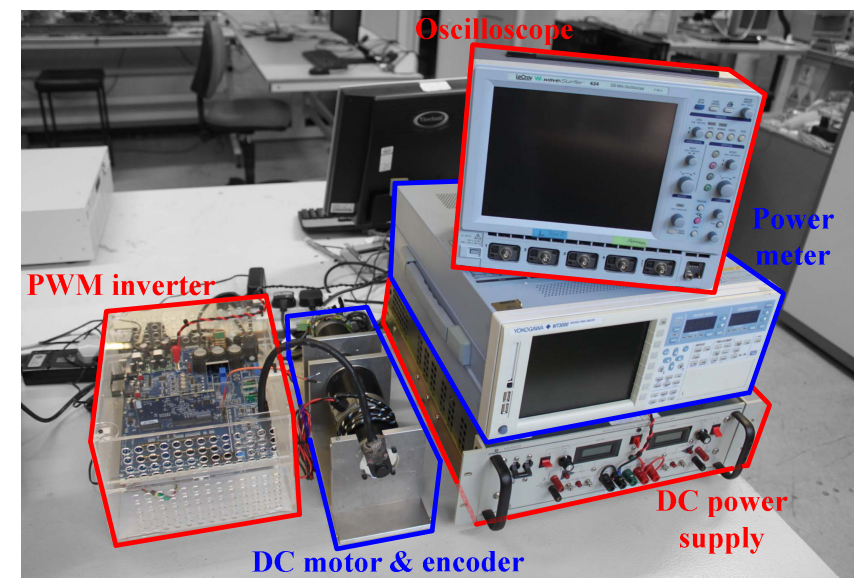

Fig. 7. Motor drive experimental setup.

analyser is adopted. It is connected in-between of DC power supply and control board for accurate comparisons. Precise positioning information is required by the proposed method so the rotor shaft is equipped with the 4000ppr incremental encoder.

Fig. 6. and Fig. 8. show the experimental results of the motor working in minimum time and minimum energy fashion. Experiments that reproduce the simulation conditions by tracking the MPC pre calculated trajectories are presented. For both cases, the motor's states follow the recalculated trajectory, but fail to reach the desired position at $0.46 \mathrm{~s}$. This happens due to the errors in motor parameters estimation and not very
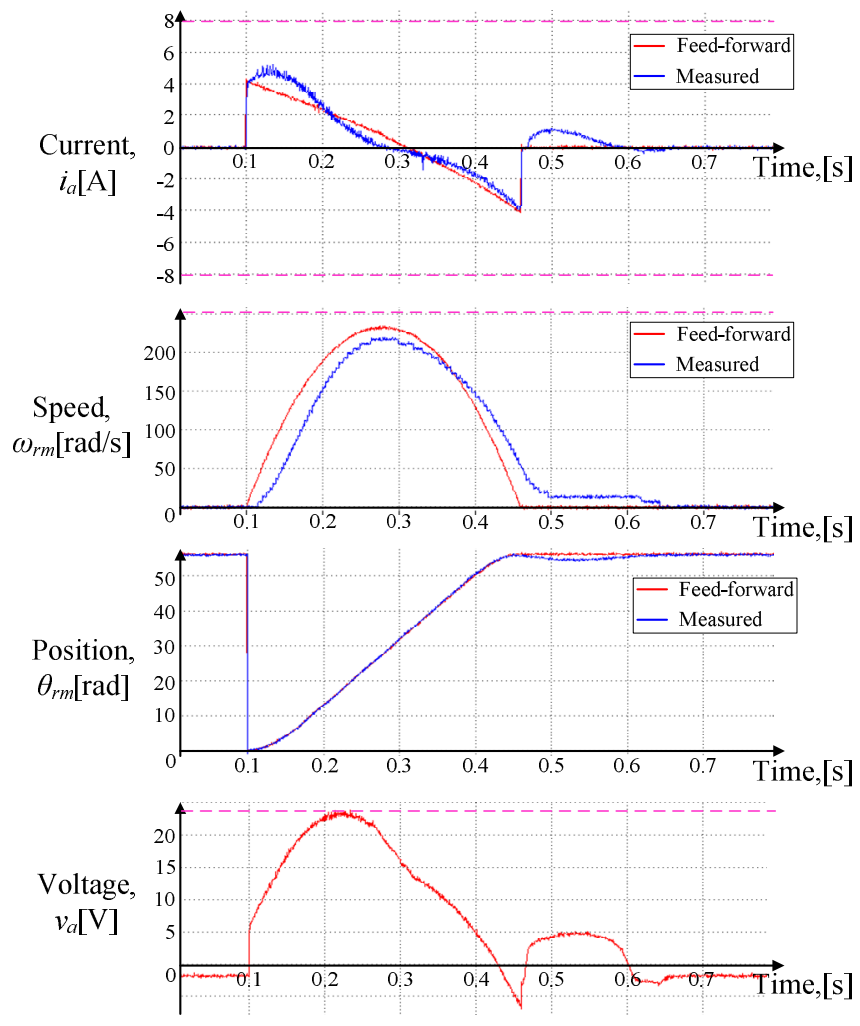

Fig. 8. Optimal energy control. 
precise cascaded controller tuning. However the same phenomena is observed on conventional G00 and G01 control and does not influence the relative energy consumption.

For the proposed method the net energy consumption after 20 cycles of repetitions by each motor was $107 \mathrm{mWh}$, whereas for $\mathrm{G} 00$ it was $123 \mathrm{mWh}$ and $116 \mathrm{mWh}$ - for G01. So the improvement is $13 \%$ compared to the former and $8 \%$ compared to the latter. These results are lower than obtained by MATLAB simulation due to the neglect of friction and inaccuracies in the motor mechanical models in the simulation.

\section{CONCLUSION}

In this paper the new control strategy for the multi-actuator system is proposed. The strategy minimizes the consumed energy, while maintaining the overall performance by minimizing the copper loss of the drive with the shorter path. The superiority of the introduced algorithm is supported by both MATLAB simulation and experiment. The method is applicable for a higher number of motors by a simple algorithm modification.

In future the cascaded control with the reference generated by MPC would be substituted with the on-line reference point tracking MPC.

\section{REFERENCES}

[1] P. Zhang, D. M. Ionel, and N. A. Demerdash, "Saliency ratio and power factor of IPM motors optimally designed for high efficiency and low cost objectives," in Energy Conversion Congress and Exposition (ECCE), 2014 IEEE, 2014, pp. 3541-3547.

[2] J. Colmenares, D. Peftitsis, G. Tolstoy, D. Sadik, H.-P. Nee, and J. Rabkowski, "High-efficiency three-phase inverter with SiC MOSFET power modules for motor-drive applications," in Energy Conversion Congress and Exposition (ECCE), 2014 IEEE, 2014, pp. 468-474.

[3] S. Mariéthoz, "Optimal control of a high efficiency low distortion 6level hybrid multilevel motor drive," in Vehicle Power and Propulsion Conference (VPPC), 2010 IEEE, 2010, pp. 1-6.
[4] M. C. Di Piazza and M. Pucci, "Techniques for efficiency improvement in PWM motor drives," Electric Power Systems Research, vol. 136, pp. 270-280, 2016

[5] D. Drury. D. Salt, "Synchronous Machine Optimal Power Control Report," 2013.

[6] J. W. Kolar, "Future Challenges for Research and Teaching in Power Electronics," presented at the First National Centre for Power Electronics Annual Conference, 2014.

[7] J. B. Dahmus and T. G. Gutowski, "An environmental analysis of machining," in ASME 2004 international mechanical engineering congress and exposition, 2004, pp. 643-652.

[8] V. A. Balogun and P. T. Mativenga, "Modelling of direct energy requirements in mechanical machining processes," Journal of Cleaner Production, vol. 41, pp. 179-186, 2013.

[9] S. D. Timar, R. T. Farouki, T. S. Smith, and C. L. Boyadjieff, "Algorithms for time-optimal control of CNC machines along curved tool paths," Robotics and Computer-Integrated Manufacturing, vol. 21, pp. 37-53, 2005.

[10] K. Erkorkmaz and Y. Altintas, "High speed CNC system design. Part I: jerk limited trajectory generation and quintic spline interpolation," International Journal of machine tools and manufacture, vol. 41, pp. 1323-1345, 2001

[11] P. Smid, Fanuc CNC Custom Macros: Industrial Press, 2005.

[12] L. Biagiotti and C. Melchiorri, Trajectory planning for automatic machines and robots: Springer Science \& Business Media, 2008.

[13] Y. Altintas, Manufacturing automation: metal cutting mechanics, machine tool vibrations, and CNC design: Cambridge university press, 2012

[14] J. B. Rawlings and D. Q. Mayne, Model predictive control: Theory and design: Nob Hill Pub., 2009.

[15] J. M. Maciejowski, Predictive control: with constraints: Pearson education, 2002

[16] L. Van den Broeck, M. Diehl, and J. Swevers, "A model predictive control approach for time optimal point-to-point motion control," Mechatronics, vol. 21, pp. 1203-1212, 2011.

[17] H.-M. Ryu and S.-K. Sul, "Position control for direct landing of elevator using time-based position pattern generation," in Industry Applications Conference, 2002. 37th IAS Annual Meeting. Conference Record of the, 2002, pp. 644-649. 